\title{
No increased risk of hepatocellular carcinoma in cirrhosis due to Wilson disease during long-term follow-up
}

\author{
Suzanne van Meer, ${ }^{*}$ Robert A de Man, ${ }^{\dagger}$ Aad $\mathrm{P}$ van den Berg, ${ }^{\ddagger}$ Roderick H J Houwen, ${ }^{\$}$ \\ Francisca H H Linn, ${ }^{\uparrow, * *,{ }^{+\dagger}}$ Martijn G H van Oijen, * Peter D Siersema* and Karel J van Erpecum* \\ Departments of *Gastroenterology and Hepatology, ${ }^{*}$ Pediatrics, and "Neurology, University Medical Center, **Rudolf Magnus Institute of \\ Neuroscience Utrecht, ${ }^{\dagger+}$ Central Military Hospital Utrecht, Utrecht, 'Department of Gastroenterology and Hepatology, Erasmus Medical Center, \\ Rotterdam, and ₹Department of Gastroenterology and Hepatology, University Medical Center, Groningen, The Netherlands
}

\author{
Key words \\ cirrhosis, hepatocellular carcinoma, Wilson \\ disease. \\ Accepted for publication 11 August 2014.

\section{Correspondence \\ Dr Karel J van Erpecum, Department of Gastroenterology and Hepatology, University Medical Center Utrecht, Po BOX 85500, 3508 GA Utrecht, The Netherlands. Email: k.j.vanerpecum@umcutrecht.nl} \\ Declaration of conflict of interest and \\ financial support \\ The authors have no supportive foundations \\ or conflicts of interest to declare.
}

\begin{abstract}
Background and Aims: Data on risk of hepatocellular carcinoma (HCC) in patients with Wilson disease are scarce. We determine HCC risk in a well-defined cohort of Wilson patients.

Methods: All patients with a confirmed diagnosis of Wilson disease (Leipzig score $\geq 4$ ) in three Dutch university referral hospitals were included in this retrospective cohort study. End of follow-up was defined as date of diagnosis of HCC, liver transplantation, death, or last available hospital visit. Also, a meta-analysis was performed to determine incidence and mortality rate of HCC in Wilson disease based on all published cohorts.

Results: In total, 130 patients with Wilson disease were followed during a median follow-up of 15 years (range 0.1-51.2). At baseline, cirrhosis was present in 74 patients ( $57 \%$ of total: $64 \%$ compensated, and $36 \%$ decompensated). At end of follow-up, liver disease severity was improved, stable or deteriorated in $20 \%, 46 \%$, and $24 \%$ of all cases (10\% unknown), respectively. Two patients developed HCC (one despite excellent decoppering after 50 years follow-up, the other with newly diagnosed Wilson disease). Estimated annual HCC risk for all patients was $0.09 \%$ (95\% confidence interval [CI]: 0.01-0.28). Subgroup analysis in cirrhotic patients revealed an annual HCC risk of $0.14 \%$ (95\% CI: $0.02-0.46)$. The meta-analysis showed an annual HCC risk of $0.04 \%$ (95\% CI: $0.01-0.10$ ) and HCC mortality rate of 2.6/10 000 person-years (95\% CI: 0.7-7.0).
\end{abstract}

Conclusions: Even in case of cirrhosis, HCC risk is low in Wilson disease. Our data do not support regular HCC surveillance in Wilson disease.

\section{Introduction}

Wilson disease (WD) is a rare autosomal recessive disorder of copper transport. Mutations in the ATP7B gene (encoding for a copper-transporting P-type ATPase (ATP7B) that is mainly expressed in hepatocytes and functions as a transmembrane copper transporter) lead to imperfect formation of holoceruloplasmin and decreased copper secretion into bile. As a result, there is accumulation of copper in the liver, brain, and other organs. Clinical presentation varies widely. In symptomatic patients, hepatic and/or neurologic disease are most common. Asymptomatic patients are most often detected by family screening.

In general, patients with cirrhosis are at significant risk for hepatocellular carcinoma (HCC). Therefore, the European Association for the Study of the Liver (EASL) guideline advises HCC surveillance by bi-annual ultrasound regardless of the underlying cause of cirrhosis. ${ }^{1}$
There is substantial evidence favoring surveillance in case of hepatitis B or C, and to a lesser extent, in case of cirrhosis due to alcohol, primary biliary cirrhosis, or hemochromatosis. ${ }^{2}$ However, strong evidence is lacking for many other cirrhotic conditions. The decision to start surveillance should depend on risk of developing HCC. Cost-effectiveness studies have indicated that HCC surveillance becomes cost-effective in patients with cirrhosis when HCC incidence exceeds $1.5 \%$ /year. ${ }^{3,4}$

Although liver cirrhosis is an important complication in patients with WD, published data on the risk of HCC in these patients are scare. In the current study, we therefore determined the risk of $\mathrm{HCC}$ in a well-defined cohort of patients with WD and related HCC risk to severity of liver disease and the efficacy of decoppering treatment.

Patients and methods. All consecutive adult patients with a diagnosis of WD in the database of the three university hospitals 
(University Medical Center Utrecht, Erasmus Medical Center Rotterdam, and University Medical Center Groningen) with referral function and caring for the large majority of WD patients in the Netherlands were included in this retrospective study. Patients generally visited the outpatient clinic at 6-month intervals, and more often if clinically indicated. Although no formal HCC surveillance was provided, radiological imaging (ultrasound, magnetic resonance imaging [MRI]) was performed often at 1-year interval in cirrhotic patients. The database does not include patients under current care of pediatricians. Diagnostic criteria for WD according to Leipzig criteria, ${ }^{5}$ endorsed by the recent EASL guideline on diagnostics and treatment of $\mathrm{WD},{ }^{6}$ were evaluated for all patients. These diagnostic criteria include the presence of Kayser-Fleischer rings, neurologic symptoms, a low ceruloplasmin level $(<0.2 \mathrm{~g} / \mathrm{L})$, Coombs-negative hemolytic anemia, an increased basal urinary copper excretion level $(>100 \mu \mathrm{g} / 24 \mathrm{~h}$ before start of decoppering therapy), increased hepatic copper concentration ( $>250 \mu \mathrm{g} / \mathrm{g}$ of dry weight), and mutations related to WD on both or one chromosome(s).

End of follow-up was defined as date of HCC diagnosis, liver transplantation, death, or last available hospital visit before June 2013. All collected data were obtained from (electronic) medical records. Diagnosis of HCC was based on EASL guideline criteria. ${ }^{1}$ Potential relation of liver disease severity or efficiency of decoppering and HCC risk was explored.

Data on initial clinical presentation (i.e. hepatic, neurologic, combined, asymptomatic) were reported for each patient. Also, data on liver disease severity were collected at the time of WD diagnosis and at the end of follow-up. As previously published, ${ }^{7}$ liver disease severity was classified as: (i) mild (disturbed liver biochemistry only); (ii) moderate (disturbed liver biochemistry combined with discrete abnormalities on ultrasound, computed tomography (CT) scan or MRI, and/or, if performed, slight or moderate fibrosis on liver biopsy but without any laboratory, radiologic, or histologic abnormalities suggesting cirrhosis); (iii) compensated end-stage liver disease (cirrhosis evident by clinical, laboratory, radiologic, or histologic findings, classified as stage A according to the Child-Pugh score); or (iv) decompensated endstage liver disease (cirrhosis evident by clinical, laboratory, radiologic, or histologic findings, classified as stage $\mathrm{B}$ or $\mathrm{C}$ according to the Child-Pugh score). At the end of follow-up, liver disease severity was classified as improved (a decrease in liver disease severity score as defined above ${ }^{7}$ from baseline), stable (liver disease severity score did not change during follow-up), or deteriorated (an increase in liver disease severity score from baseline). Performance of liver transplantation was also reported.

For each patient, data regarding treatment for WD during the entire follow-up were obtained. In patients who were treated for at least 1 year, efficacy of decoppering was determined at the end of follow-up based on values of serum non-ceruloplasmin-bound copper concentration and 24-h-urinary copper excretion. Efficacy of decoppering was classified as: (i) excellent (serum nonceruloplasmin-bound copper concentration $<10 \mu \mathrm{g} / \mathrm{dL}$ and $24-\mathrm{h}$ urinary copper excretion $<100 \mu \mathrm{g}$ ); (ii) moderate (serum nonceruloplasmin-bound copper concentration $<10 \mu \mathrm{g} / \mathrm{dL}$ or $24-\mathrm{h}$ urinary copper excretion $<100 \mu \mathrm{g}$ ); or (iii) poor (serum non-ceruloplasmin-bound copper concentration $>10 \mu \mathrm{g} / \mathrm{dL}$ and 24-h urinary copper excretion $>100 \mu \mathrm{g}$ ). In case of treatment with chelators, 24-h-urinary copper excretion was determined with continued chelator treatment and should not exceed $500 \mu \mathrm{g}$ to be qualified as appropriate. ${ }^{7}$ When urine aliquots were used for follow-up data on urinary copper excretion, we estimated 24-h urinary copper excretion, assuming 24-h urinary volumes of $1500 \mathrm{~mL}$.

Furthermore, a comprehensive search of the literature was performed to identify studies that investigated long-term outcomes, including occurrence and/or mortality of HCC, in patients with WD. The electronic database of PubMed was searched for articles published in the English language. The search term comprised synonyms for "Wilson disease" as domain and "hepatocellular carcinoma" or "long-term follow up" as outcome. Studies were eligible for inclusion if they investigated long-term outcomes of WD patients and reported total number of patients that developed or died from HCC and person-years of follow-up. When total number of person-years was not mentioned, it was estimated, if possible, based on mean duration of follow-up and number of patients. In order to evaluate whether our search included all published studies, we checked the reference lists of the included articles.

Statistical analysis. Values are given as means and ranges or in case of a non-Gaussian distribution, as medians and ranges. Differences in values between two time points (i.e. baseline and end of follow-up) and between subgroups were analyzed for significance with Pearson's Chi-square test or Fisher's Exact test, as appropriate. A two-sided $P$-value $<0.05$ was considered statistically significant. A sample size weighted pooled analysis, based on data of the included studies and the current report, was conducted to estimate annual HCC risk and HCC mortality rate in patients with WD. Estimated HCC incidences and mortality rates for the total group, the subgroup of cirrhotics, and the data of the metaanalysis were calculated with the Mid-P exact test based on the total number of patients who developed or died from HCC and person-years of follow-up.

\section{Results}

Baseline characteristics. A total of 130 patients with WD were identified. Baseline characteristics are given in Table 1. More than half of all patients displayed Kayser-Fleischer rings at baseline $(59 \%)$. Patients with neurologic symptoms were more likely to have Kayser-Fleischer rings than patients with exclusive hepatic presentation ( $88 \%$ vs $43 \%$ ). Serum ceruloplasmin levels were reduced in $82 \%$ of all patients, whereas $24-\mathrm{h}$ urinary copper excretion was elevated in $72 \%$ of all patients. Coombs-negative hemolytic anemia was present in $19 \%$ of patients at the time of WD diagnosis. Quantitative hepatic copper content was determined in 37 patients. The median copper content was $652 \mu \mathrm{g} / \mathrm{g}$ dry liver weight (range $44-1600 \mu \mathrm{g} / \mathrm{g}$ ). In 32 of these patients $(86 \%$ ), hepatic copper content was above $250 \mu \mathrm{g} / \mathrm{g}$. Mutations related to WD were detected on both or one chromosome(s) in 68 (90\%) and $5(5 \%)$ of the 76 patients in whom mutation analysis was performed. The p.H1069Q mutation was present in 44 patients $(58 \%$ : $18 \%$ homozygous and $40 \%$ heterozygous, respectively). Overall, median Leipzig score was 8 points (range 4-13).

Median age at the time of diagnosis of WD was 16 years (range 0-43). In two infant patients, WD diagnosis was based on 
Table 1 Baseline characteristics of 130 patients with Wilson disease

\begin{tabular}{lc}
\hline Patient no & 130 \\
\hline Presence of diagnostic criteria of & \\
$\quad$ Wilson disease ( $[$ [\%]) & $77(59)$ \\
Kayser-Fleischer rings & $50(38)$ \\
Neurologic symptoms & $106(82)$ \\
Serum ceruloplasmin < 0.2 g/L & $25(19)$ \\
Coombs-negative hemolytic anemia & $94(72)$ \\
24-h urinary copper excretion > 100 $\mu g$ & $32 / 37(86)^{\dagger}$ \\
Liver biopsy (Cu > 250 $\mu$ g/g dry weight) & $68 / 76(90)^{\dagger}$ \\
Positive genetic testing & $5 / 76(5)^{\dagger}$ \\
$\quad$ Mutation(s) on both chromosomes & $8(4-13)$ \\
$\quad$ Mutation(s) on 1 chromosome & $16(0-43)$ \\
Leipzig score (median and range) & $65(50)$ \\
Age at diagnosis (median and range, in years) & \\
Male gender ( $[\%]$ ) & $72(55)$ \\
Presentation ( $[\%]$ ) & $11(9)$ \\
Hepatic & $39(30)$ \\
Neurologic & $5(4)$ \\
Combination & $3(2)$ \\
Asymptomatic & $14.8(0.1-51.2)$ \\
Unknown & \\
\hline
\end{tabular}

${ }^{\dagger}$ Percentages are calculated based on number of total patients in whom quantitative hepatic copper content was determined $(n=37)$, respectively mutation analysis was performed ( $n=76)$.

Results indicate numbers and, between brackets, percentages.

mutations related to WD on both chromosomes. Male to female ratio was nearly equal. Presentation was exclusively hepatic in more than half of the patients (55\%). Almost one third of patients $(30 \%)$ had both neurologic and hepatic presentation at the time of diagnosis of $\mathrm{WD}$, whereas a small percentage of patients presented with only neurologic symptoms (9\%) or were asymptomatic (4\%). In three patients, baseline data were not available, and mode of presentation was unknown. Median duration from WD diagnosis to end of follow-up was 15 years (range 0.1-51.2 years) (Table 1). Total follow-up duration in this study was 2336 patient years.

Liver disease severity. At baseline, cirrhosis was present in 74 patients (57\% of total: $64 \%$ compensated and $36 \%$ decompensated). Distribution of severity of liver disease changed significantly over time $(P=0.009)$, mainly due to progression from compensated to decompensated cirrhosis (Table 2). At the end of follow-up, liver disease severity was improved, stable and deteriorated in $20 \%, 46 \%$, and $24 \%$ of patients, respectively. Data on liver disease severity were incomplete in 13 patients (10\%). Twentyeight patients (22\% of all patients) received a liver transplant. Liver transplantation was performed within 1 month of WD diagnosis in seven cases. Overall, median duration from WD diagnosis to liver transplantation was 1.4 years (range $0.1-38.5$ years). Five patients died due to complications of their liver disease, and two deaths were related to liver transplantation.

Treatment and efficiency of decoppering. For patients who were treated for at least 1 year $(n=111)$, prescribed medication for WD during the entire disease course and efficiency
Table 2 Severity of liver disease ${ }^{7}$ at baseline and end of follow-up in 130 patients with Wilson disease

\begin{tabular}{|c|c|c|c|}
\hline & At baseline & At end of follow-up & $P$-value \\
\hline & $n(\%)$ & $n(\%)$ & 0.009 \\
\hline Normal & $12(9)$ & $20(15)$ & \\
\hline Mild & $10(8)$ & $12(9)$ & \\
\hline Moderate & $23(18)$ & $28(22)$ & \\
\hline Compensated cirrhosis & $47(36)$ & $29(22)$ & \\
\hline Decompensated cirrhosis & $27(21)$ & $39(30)^{\dagger}$ & \\
\hline Unknown & $11(8)$ & $2(2)$ & \\
\hline
\end{tabular}

Table 3 Efficiency of decoppering during treatment in 130 patients with Wilson disease

\begin{tabular}{lr}
\hline & $n(\%)^{\dagger}$ \\
\hline Treatment $^{\ddagger}$ & $102(92)$ \\
Zinc & $77(69)$ \\
Penicillamine & $16(14)$ \\
$\quad$ Trientine & \\
Efficiency of decoppering at end of follow-up & $38(34)$ \\
$\quad$ Excellent & $47(42)$ \\
Moderate & $14(13)$ \\
Poor & $12(11)$ \\
Unknown & $17.1(1.5-51.2)$ \\
Duration of follow-up (median and range, in years) &
\end{tabular}

${ }^{\top}$ Total number of patients is 111 (only patients with $\geq 1$ year therapy included)

${ }^{\ddagger}$ Alone, sequentially or combined.

of decoppering at the end of follow-up are given in Table 3. Median duration of follow-up in this group of patients was 17.1 years (range 1.5-51.2 years). Zinc was frequently prescribed, with $92 \%$ of patients being treated with zinc alone, sequentially or combined with chelators. Penicillamine treatment was initially started in $59 \%$ of patients, whereas $69 \%$ received penicillamine at any time during the disease course. Trientine was prescribed in $14 \%$ of patients. Efficiency of decoppering was determined at the end of follow-up based on values of serum non-ceruloplasminbound copper concentration and 24-h urinary copper excretion. Excellent decoppering was present in $34 \%$ of patients. Nevertheless, most patients (42\%) had moderate efficiency of decoppering. In $13 \%$ of patients efficiency was poor, and in $11 \%$ data on serum non-ceruloplasmin-bound copper concentration copper and 24-h urinary copper excretion at the end of follow-up were not available.

HCC development. Two patients were diagnosed with HCC. The first HCC case occurred in a 39-year-old male patient. He presented with decompensated cirrhosis in combination with HCC (tumor size: $6 \mathrm{~cm}$, Barcelona Clinic Liver Cancer [BCLC] stage D, alpha-fetoprotein [AFP] level: $2295 \mu \mathrm{g} / \mathrm{L}$ ). There were no additional risk factors for $\mathrm{HCC}$ development, such as other metabolic liver diseases, viral hepatitis, or alcohol abuse. WD diagnosis was based on the presence of Kayser-Fleischer rings, a low 
Table 4 Details of studies investigating long-term follow-up, including occurrence or mortality of hepatocellular carcinoma, in patients with Wilson disease

\begin{tabular}{llll}
\hline First author (year) & $\begin{array}{l}\text { Follow-up time } \\
\text { (person-years) }\end{array}$ & $\begin{array}{l}\text { No. of patients } \\
\text { who developed } \\
\text { HCC }\end{array}$ & $\begin{array}{l}\text { No. of patients } \\
\text { who died of } \\
\text { HCC }\end{array}$ \\
\hline Beinhardt $(2014)^{8}$ & 3116 & 0 & 0 \\
Bruha $(2011)^{9}$ & $1761^{\dagger}$ & - & 1 \\
Merle $(2007)^{12}$ & 2476 & 0 & 0 \\
Svetel $(2009)^{13}$ & $1576^{\dagger}$ & - & 0 \\
${\text { Lowette }(2010)^{15}}^{375^{\dagger}}$ & 1 & 0 \\
Current report & 2336 & 2 & 2 \\
\hline
\end{tabular}

${ }^{\dagger}$ Calculated based on mean follow-up and number of patients.

HCC, hepatocellular carcinoma.

ceruloplasmin level $(0.13 \mathrm{~g} / \mathrm{L})$, increased 24-h urinary copper excretion $(584 \mu \mathrm{g})$, and mutations related to WD on both chromosomes (Leipzig score: 9 points). Treatment for HCC was not performed. He died 4 months after presentation. The second patient was a 63-year-old female with unequivocal WD diagnosed 50 years earlier. WD diagnosis was based on the presence of Kayser-Fleischer rings, a low ceruloplasmin level (0.05 g/L), increased $24-\mathrm{h}$ urinary copper excretion $(345 \mu \mathrm{g})$, and mutations related to WD on both chromosomes (Leipzig score: 10 points). Despite excellent decoppering at the end of follow-up after initial penicillamine treatment and subsequent maintenance therapy with zinc acetate (serum non-ceruloplasmin-bound copper: $<10 \mu \mathrm{g} / \mathrm{dL}$ and $24-\mathrm{h}$ urinary copper excretion: $34 \mu \mathrm{g}$ ), she progressed to decompensated cirrhosis in which an HCC developed (BCLC stage D). AFP level at time of diagnosis was $120 \mu \mathrm{g} / \mathrm{L}$. No additional risk factors for liver disease were present in this patient. Treatment for HCC was not performed. She died 6 months after HCC diagnosis.

Estimated annual HCC risk for all patients was 0.09\% (95\% confidence interval [CI]: 0.01-0.28). Subgroup analysis in cirrhotic patients indicated an annual HCC risk of $0.14 \%$ (95\% CI: 0.02-0.46). Both patients died due to their HCC, and therefore incidence and mortality rates did not differ (8.6/10 000 personsyears for all patients and 13.9/10 000 persons-years in cirrhotic patients, respectively).

Our literature search resulted in the identification of nine other studies that investigated outcomes, including $\mathrm{HCC}$ occurrence, in WD patients during long-term follow-up. ${ }^{8-16}$ After exclusion of four studies because of missing data on duration of followup, ${ }^{10,11,14,16,17}$ data of the current report and five other studies $^{8,9,12,13,15}$ were included in the meta-analysis. Study characteristics and outcomes of the included studies are given in Table 4. Based on data of the current study and three other studies, ${ }^{8,12,15}$ the pooled annual HCC risk was $0.04 \%$ (95\% CI: 0.01-0.10). Based on data of the current study and five other studies that reported HCC mortality, ${ }^{1,8,9,12-15}$ the pooled mortality rate was 2.6/10 000 person-years (95\% CI: 0.7-7.0).

\section{Discussion}

In the EASL guideline, HCC surveillance is generally recommended in all patients with cirrhosis, regardless of underlying cause. ${ }^{1}$ Nevertheless, it remains to be seen whether this approach is cost-effective. The main finding of the current study is that even with advanced cirrhosis, HCC risk appears to be low in patients with WD.

Previous publications on HCC risk in WD are limited. The review by Thattil et al ${ }^{18}$ summarizes 28 published case reports. Almost all HCC cases occurred in cirrhotic livers with a predominance of males. Walshe $e t a l^{11}$ performed a retrospective study to assess the frequency of abdominal malignancies in general in WD. Two cohorts of patients $(n=363)$ from two specialist WD clinics (Cambridge/London: $n=310$ in the period: 1955-2000 and Uppsala: $n=53$ in the period: 1966-2002) were included in this study. Of all patients who were followed for 10 years or more $(n=159)$, nine patients developed abdominal malignancies (including two HCCs). Incidences rates of hepatic, biliary, and pancreatic cancers were claimed to be significantly higher in the study population than in the general population of Sweden in 2000. Detailed information on WD diagnosis and treatment was not provided for this cohort, and other coexistent causes of liver disease were not rigorously excluded, although patients came from diverse locations including Egypt, Greece, Serbia, Italy, and the UK. Furthermore, several other studies describing clinical presentation and outcomes of large cohorts of WD patients from Austria, Czech, Denmark, and Germany mentioned only one or none HCC case(s) during long-term follow-up. ${ }^{8-10,12}$

Based on our findings, annual HCC incidence in WD patients $(0.09 \%$ and $0.14 \%$ in all patients and with cirrhosis, respectively) is far below the accepted threshold incidence for HCC surveillance to be cost-effective (1.5\%/year for patients with cirrhosis). ${ }^{3,4}$ Results of our meta-analysis confirmed the low HCC incidence as well as HCC-related mortality rate in WD patients. Therefore, our data do not support regular surveillance in patients with WD, even in case of cirrhosis, unless additional risk factors for HCC (e.g. hepatitis B or C, alcohol) are present. Additionally, none of our patients developed a cholangiocarcinoma, despite the increased risk for this tumor in cirrhotic livers in general.

The reason for the low HCC incidence in WD patients compared to other chronic liver diseases remains unclear. Based on earlier animal studies, it has previously been suggested that excessive copper accumulation might have a protective effect on hepatocarcinogenesis. ${ }^{19-22}$ On the other hand, in the Long-Evans Cinnamon rat model for WD, long and persistent copper accumulation resulted in an increased risk of HCC which could be prevented by administration of D-penicillamine. ${ }^{23}$ In this model, carcinogenesis is thought to be the result of liver injury leading to chronic inflammation and cirrhosis due to chronic copper accumulation. Relation between HCC risk and efficacy of decoppering treatment could not be analyzed in detail in the current study due to the relatively small number of HCC cases. Nevertheless, most WD patients exhibited moderate or poor decoppering without developing HCC. Also, the second HCC case occurred in a patient despite excellent decoppering.

Our study has some strengths and limitations. Most important, a diagnosis of WD was unequivocally established in all cases (Leipzig score $\geq 4$ ), and detailed information on WD treatment as well as effectiveness of decoppering was available. Also, the long follow-up (median 15 years: up to 51 years) in this cohort allowed a reasonable reliable estimation of HCC risk. On the other hand, the study design is retrospective. Moreover, patients could have 
been missed as a result of death before diagnosis of HCC or WD. Nevertheless, our results are in accordance with the clinical observation that $\mathrm{HCC}$ in patients with WD is rare. This contention is supported by the fact that during the period 2003-2013, no liver transplant was performed in the Netherlands for HCC in WD patients. In this period, nine liver transplantations were performed in WD patients with decompensated liver cirrhosis, all without $\mathrm{HCC}$ at pathological examination of the explant. The total number of transplantations for HCC in the Netherlands was 165 in this period (data from the Eurotransplant database). Furthermore, majority of WD patients in the Netherlands is treated in one of the three university hospitals that participated in this study.

In conclusion, we found that the risk of HCC development in WD patients on long-term follow-up was low. Our data therefore do not support regular HCC surveillance in patients with WD in absence of other risk factors.

\section{References}

1 European Association for the Study of the Liver, European Organisation for the Research and Treatment of Cancer. EASL-EORTC clinical practice guidelines: management of hepatocellular carcinoma. J. Hepatol. 2012; 56: 908-43.

2 van Meer S, de Man RA, Siersema PD, van Erpecum KJ. Surveillance for hepatocellular carcinoma in chronic liver disease: evidence and controversies. World J. Gastroenterol. 2013; 19: 6744-56.

3 Sarasin FP, Giostra E, Hadengue A. Cost-effectiveness of screening for detection of small hepatocellular carcinoma in western patients with Child-Pugh class A cirrhosis. Am. J. Med. 1996; 101: 422-34.

4 Arguedas MR, Chen VK, Eloubeidi MA, Fallon MB. Screening for hepatocellular carcinoma in patients with hepatitis $\mathrm{C}$ cirrhosis: a cost-utility analysis. Am. J. Gastroenterol. 2003; 98: 679-90.

5 Ferenci P, Caca K, Loudianos G et al. Diagnosis and phenotypic classification of Wilson disease. Liver Int. 2003; 23: 139-42.

6 European Association for the Study of the Liver. EASL Clinical Practice Guidelines: Wilson's disease. J. Hepatol. 2012; 56: 671-85.

7 Linn FH, Houwen RH, van Hattum J, van der Kleij S, van Erpecum KJ. Long-term exclusive zinc monotherapy in symptomatic Wilson disease: experience in 17 patients. Hepatology 2009; 50: 1442-52.

8 Beinhardt S, Leiss W, Stattermayer AF et al. Long-term outcomes of patients with Wilson disease in a large Austrian cohort. Clin. Gastroenterol. Hepatol. 2014; 12: 683-9.
9 Bruha R, Marecek Z, Pospisilova L et al. Long-term follow-up of Wilson disease: natural history, treatment, mutations analysis and phenotypic correlation. Liver Int. 2011; 31: 83-91.

10 Moller LB, Horn N, Jeppesen TD et al. Clinical presentation and mutations in Danish patients with Wilson disease. Eur. J. Hum. Genet. 2011; 19: 935-41.

11 Walshe JM, Waldenstrom E, Sams V, Nordlinder H, Westermark K. Abdominal malignancies in patients with Wilson's disease. QJM 2003; 96: 657-62.

12 Merle U, Schaefer M, Ferenci P, Stremmel W. Clinical presentation, diagnosis and long-term outcome of Wilson's disease: a cohort study. Gut 2007; 56: 115-20.

13 Svetel M, Pekmezovic T, Petrovic I et al. Long-term outcome in Serbian patients with Wilson disease. Eur. J. Neurol. 2009; 16: $852-7$.

14 Czlonkowska A, Tarnacka B, Litwin T, Gajda J, Rodo M. Wilson's disease-cause of mortality in 164 patients during 1992-2003 observation period. J. Neurol. 2005; 252: 698-703.

15 Lowette KF, Desmet K, Witters P et al. Wilson's disease: long-term follow-up of a cohort of 24 patients treated with D-penicillamine. Eur. J. Gastroenterol. Hepatol. 2010; 22: 564-71.

16 Kumagi T, Horiike N, Michitaka K et al. Recent clinical features of Wilson's disease with hepatic presentation. J. Gastroenterol. 2004; 39: 1165-9.

17 de Bem RS, Muzzillo DA, Deguti MM, Barbosa ER, Werneck LC, Teive HA. Wilson's disease in southern Brazil: a 40-year follow-up study. Clinics (Sao Paulo) 2011; 66: 411-6.

18 Thattil R, Dufour JF. Hepatocellular carcinoma in a non-cirrhotic patient with Wilson's disease. World J. Gastroenterol. 2013; 19: $2110-3$.

19 Howell JS. The effect of copper acetate on p-dimethylaminoazobenzene carcinogenesis in the rat. Br. J. Cancer 1958; 12: 594-608.

20 King H, Spain J, Clayton C. Dietary copper salts and azo dye carcinogenesis. J. Nutr. 1957; 63: 301-9.

21 Kamamoto Y, Makiura S, Sugihara S, Hiasa Y, Arai M. The inhibitory effect of copper on DL-ethionine carcinogenesis in rats. Cancer Res. 1973; 33: 1129-35.

22 Wilkinson ML, Portmann B, Williams R. Wilson's disease and hepatocellular carcinoma: possible protective role of copper. Gut 1983; 24: 767-71.

23 Jong-Hon K, Togashi Y, Kasai H, Hosokawa M, Takeichi N. Prevention of spontaneous hepatocellular carcinoma in Long-Evans cinnamon rats with hereditary hepatitis by the administration of D-penicillamine. Hepatology 1993; 18: 614-20. 